\title{
Time-Domain Neural Network Receiver for Nonlinear Frequency Division Multiplexed
} Systems

Jones, Rasmus Thomas; Gaiarin, Simone; Yankov, Metodi Plamenov; Zibar, Darko

Published in:

I E E E Photonics Technology Letters

Link to article, DOI:

10.1109/LPT.2018.2831693

Publication date:

2018

Document Version

Peer reviewed version

Link back to DTU Orbit

Citation $(A P A)$ :

Jones, R. T., Gaiarin, S., Yankov, M. P., \& Zibar, D. (2018). Time-Domain Neural Network Receiver for Nonlinear Frequency Division Multiplexed Systems. I E E E Photonics Technology Letters, 30(12), 1079 - 1082. https://doi.org/10.1109/LPT.2018.2831693

\section{General rights}

Copyright and moral rights for the publications made accessible in the public portal are retained by the authors and/or other copyright owners and it is a condition of accessing publications that users recognise and abide by the legal requirements associated with these rights.

- Users may download and print one copy of any publication from the public portal for the purpose of private study or research.

- You may not further distribute the material or use it for any profit-making activity or commercial gain

- You may freely distribute the URL identifying the publication in the public portal 


\title{
Time-Domain Neural Network Receiver for Nonlinear Frequency Division Multiplexed Systems
}

\author{
Rasmus T. Jones, Simone Gaiarin, Metodi P. Yankov, and Darko Zibar
}

\begin{abstract}
The nonlinear Fourier transform is a new approach of addressing the capacity limiting Kerr nonlinearities in optical communication systems. It exploits the property of integrability of the lossless nonlinear Schrödinger equation and thus incorporates nonlinearities as an element of the transmission. However, practical links employing erbium-doped fiber amplifiers include losses/gains and introduce noise which breaks the integrability of the nonlinear Schrödinger equation. Although the lossless path average approximation proposes an integrable model, its imprecision still leads to unintended distortions and thus performance degradation. We propose an alternative receiver for nonlinear frequency division multiplexing optical communication systems using techniques from machine learning. It is highly adaptive as it learns from previously transmitted pulses and thus holds no assumptions on the system and noise distribution. The detection method presented is fully applied in time-domain and omits the nonlinear Fourier transform. The numerical results provide a benchmark for nonlinear Fourier transform based detection of high order solitons for fiber links with losses and noise present.
\end{abstract}

Index Terms-optical fiber communication, coherent communication, machine learning, (inverse) nonlinear Fourier transform

\section{INTRODUCTION}

Kerr nonlinearities are limiting the data throughput of coherent optical communication systems, while the demand for data driven applications is increasing. The optical fiber research community is urged for new approaches to overcome the imminent capacity shortcoming [1]. A promising and emerging mathematical method, the nonlinear Fourier transform (NFT), provides an entirely new way of transmitting data in optical communication systems $[2,3]$. Communication systems based on the NFT integrate Kerr nonlinearities by exploiting the integrability of the nonlinear Schrödinger equation (NLSE). The NFT associates a so-called nonlinear spectrum, composed of a discrete and continuous part, to a signal. The evolution of the spectrum upon spatial propagation in the nonlinear fiber channel is described by a linear transformation [4]. This property and the inverse linear transformation allows to recover the transmitted data if encoded in the nonlinear spectrum. A common communication scheme employing the NFT is known as nonlinear frequency division multiplexing (NFDM) [5]. In this work, we use NFDM with information only carried by the discrete spectrum as complex eigenvalues and their spectral amplitudes. In the time-domain those complex eigenvalues correspond to higher order solitons. However, fiber losses and noise from optical amplifiers in real systems conflict with the

R.T. Jones, S. Gaiarin, M.P. Yankov and D. Zibar are with the Department of Photonics Engineering, Technical University of Denmark, 2800 Kgs. Lyngby, Denmark, e-mail: rajo@fotonik.dtu.dk

Manuscript received January XX, XXXX; revised January XX, XXXX. assumption of integrability of the NLSE which leads to an unintended nonlinear evolution of the spectrum. Therefore, robust ways of countering noise and loss in NFT systems are of high research interest. Methods averaging the effect of fiber loss leading to an integrable model [6], and decoding schemes [7] have been proposed to deal with the impact of losses and noise. Yet, the propagation is still sub-optimal and an optimal receiver for NFT based transmission in practical channels is unknown. An optimal NFT receiver must consider how distortions to the time-domain signal are translated into the nonlinear spectral domain as described in [4] and references therein.

We propose a novel machine learning based receiver, that maps from time-domain solitons directly to symbol decisions. Trained on previous transmissions, the distortion characteristics are learned and applied for inference for future decisions. It is trained using a neural network (NN) [8], entirely omitting the NFT at the receiver. It is shown that a trained NN recovers the data encoded on solitons in presence of losses and noise. It significantly outperforms the NFT based detection for a practical link configuration, leading to an improved bit error rate (BER). For comparison, a simpler receiver is trained, learning the distortion average and using the Euclidean minimum distance (MD) for detection [9]. The MD receiver is also outperformed by the $\mathrm{NN}$ which indicates that the accummulated distortion of the system is not i.i.d. Gaussian distributed [10].

This letter is structured as follows. In Section 2, the basic concept of an NFDM comunication system and the time-domain receivers of this letter are presented. Section 3 describes the numerical simulation model of the fiberoptic communication link. Different NN configurations are tested for different transmission lengths and optimized for two transmission lengths, leading to numerical simulation results of the system performance in BER. The results are discussed and concluded thereafter in Sections 4 and 5.

\section{Methods}

\section{A. NFDM communication system}

The normalized NLSE

$$
i \frac{\partial q(t, z)}{\partial z}=\frac{\partial^{2} q(t, z)}{\partial t^{2}}+2|q(t, z)|^{2} q(t, z),
$$

provides the mathematical foundation for NFDM communication systems. The NFDM based transmitter generates a time-domain waveform of truncated and thus non overlapping soliton pulses

$$
A(t, z=0)=\sum_{k} u_{k}\left(t-k T_{s}, z\right),
$$


(a)

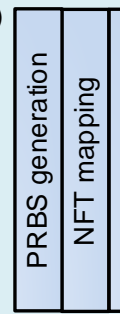

(b)

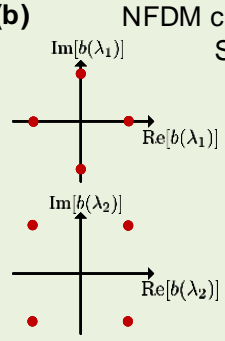

Tx Transmission link

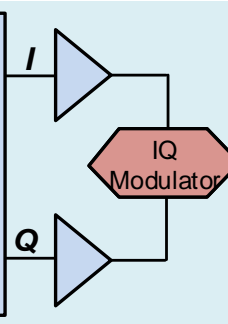
Soliton
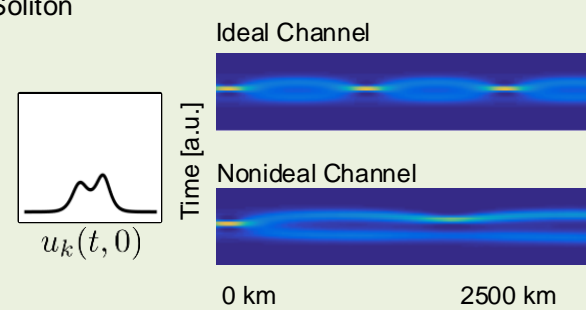
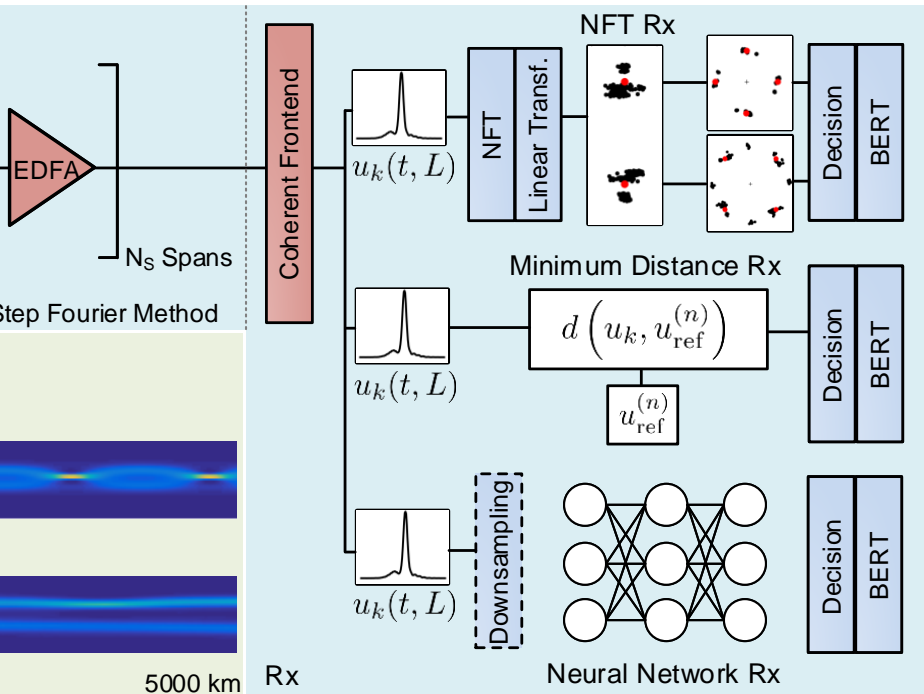

Fig. 1. Simulation setup (a) (transmitter $T x$, transmission link and receiver $R x$ ), and evolution of one soliton pulse (b) through an ideal (lossless and noiseless) and nonideal realistic channel.

where each soliton $u_{k}(t, z)$ is an NFDM symbol and $T_{s}$ is the duration of the symbol period in which a single pulse lies. In the nonlinear spectral domain an NFDM symbol is represented by QPSK modulated scattering coefficients, i.e. $b\left(\lambda_{i}\right)$ for single and $\left\{b_{1}\left(\lambda_{i}\right), b_{2}\left(\lambda_{i}\right)\right\}$ for dual polarization [11], associated to the eigenvalues $\lambda_{i}, i=1,2$. Thus, NFDM constellations in the nonlinear spectrum are mapped to soliton pulses in timedomain with the inverse NFT using the lossless path average approximation [6], which leads to $N$ distinguishable pulses,

$$
N=M^{(p \cdot E)},
$$

where $E$ is the number of eigenvalues, $M$ the order of the constellation, and $p$ the number of polarizations. After transmission and coherent detection, the received pulses are transformed back into the nonlinear spectrum using the NFT. After a linear shift the transmitted scattering coefficients are recovered, as shown in Fig. 1.

\section{B. Machine learning receivers}

In this paper, three detection schemes of NFDM communication systems are analysed. The first scheme employs a standard NFT based detection. The second and third schemes are applied directly in time-domain and using the Euclidean $\mathrm{MD}$ and an NN, respectively, as shown in Fig. 1. After transmitting over a distance $L$, the MD receiver is trained by averaging over multiple received instances of each of the $N$ individual solitons. These averages are used as reference $u_{\text {ref }}^{(n)}(t, L), n=1 . . N$, to detect future received solitons. Hence, for every received soliton pulse $u_{k}(t, L)$ the Euclidean distance to all references is calculated and the reference pulse with minimum distance is chosen for decoding:

$$
n_{\mathrm{opt}}[k]=\underset{n}{\operatorname{argmin}}\left(\int_{-\infty}^{\infty}\left|u_{k}(t, L)-u_{\mathrm{ref}}^{(n)}(t, L)\right|^{2} d t\right),
$$

where the integral becomes a summation for sampled signals. The MD receiver does not take the noise distribution into account, whereas an NN does, i.e., the NN receiver learns a near optimal probability distribution of the received symbols during the training process. With a sampling rate of $R$ samples per symbol (SPS), all received pulses lie in an $R p$ dimensional complex space. Thus, the NN learns the mapping from the $R p$ dimensional complex space of pulses to a decision on one of the $N$ possible transmitted symbols. The $R p$ dimensional complex input to the $\mathrm{NN}$ is separated in real and imaginary parts and the decisions are transformed to one-hot encoded vectors of length $N$ [8]. Thus, the $\mathrm{NN}$ has $2 R p$ input units and $N$ output units.

\section{Simulation SEtup}

The described detection schemes are applied in a simulation. The setup is described in Fig. 1 (a). The transmitter consists of a pseudorandom binary sequence (PRBS) pattern generator, an NFDM symbol mapper and an implementation of the inverse NFT algorithm to map symbols to soliton pulses. Single and dual polarization NFDM systems are simulated. Both deploying $E=2$ eigenvalues $\left(\lambda_{1}=0.3 \mathrm{j}\right.$ and $\left.\lambda_{2}=0.6 \mathrm{j}\right)$ and QPSK constellations with unitary radius ( $M=4$ ), resulting in $N=16$ and $N=256$ symbols for single and dual polarization, respectively. A rotational offset of $\pi / 4$ is applied to the constellations associated to the eigenvalue with larger imaginary part, as depicted in Fig. 1 (b). The symbol rate is $1 / T_{s}=1 \mathrm{GBd}$. The electrical signal drives a Mach-Zehnder modulator in pseudo-linear region. An ideal laser is considered, neglecting the effect of phase noise. The waveform is propagated using the split-step Fourier method with dispersion parameter $17.5 \mathrm{ps} /(\mathrm{nm} \mathrm{km})$, nonlinear coefficient $1.25 \mathrm{1} /(\mathrm{W} \mathrm{km})$, fiber loss $0.195 \mathrm{~dB} / \mathrm{km}$ and increasing transmission distance from 0 to $5004 \mathrm{~km}$. The link is divided into $N_{s}$ spans of $41.7 \mathrm{~km}$ each interleaved by an erbium-doped fiber amplifier (EDFA). After coherent detection the waveform is sliced into single pulses at $R=128$ samples 
and passed on to the three receiver schemes for detection. The MD receiver is trained with 100,000 training symbols to extract its references. The NN receiver has one hidden layer with 32 and 128 hidden units for single and dual polarization. All NNs are trained with sigmoid activation functions, softmax output layer, cross-entropy loss function, stochastic gradient descent and the Adam optimization algorithm [8]. The training data size is 100,000 symbols for each distance, where $90 \%$ are used for the actual training and $10 \%$ for testing. To avoid over-fitting, the training is stopped when the test performance ceases to improve (early stopping) [8]. An independent data set of 100,000 symbols for each distance is used for validation and all performance estimations in this paper. This makes BERs estimated above $1.25 \cdot 10^{-4}\left(6.25 \cdot 10^{-5}\right)$ reliable since at least 100 errors are seen for the $800,000(1,600,000)$ transmitted bits for the single (dual) polarization case. For further analysis of the hyperparameters, the training data size, the number of hidden units and the number of SPS are swept.

\section{RESUlTS \& DiscUSSION}

\section{A. Temporal/Spectral Evolution and BER performance}

For correct detection it is important that the solitons remain stable throughout transmission. This means that the solitons stay temporally confined in a time slot no larger than the transmitter slot $T_{s}$. In order to demonstrate how the power envelope of the NFDM generated solitons evolve during transmission, one instance of the possible solitons is propagated under lossless and noiseless (ideal) conditions and with losses and noise (nonideal). In Fig. 1 (b), it is observable that under ideal conditions (top) the soliton breathes but stays stable, but under nonideal conditions (bottom) the soliton distorts and the pulse is in the process of splitting. When a pulse splits and disperses out of its time slot the carried information is lost. Further, to demonstrate how the spectral density of NFDM solitons evolve during transmission, a whole train of soliton pulses is propagated under nonideal conditions and the evolution of the baseband frequency spectrum is shown in Fig. 2 along with the bandwidth containing 99\% of the power. It is observable that the bandwidth changes with the transmission distance and thus the receiver sampling rate must be chosen accordingly (further discussed in Section IV-C). For performance estimation, all three detection schemes are simulated. In Fig. 3, BER performances for single (a) and

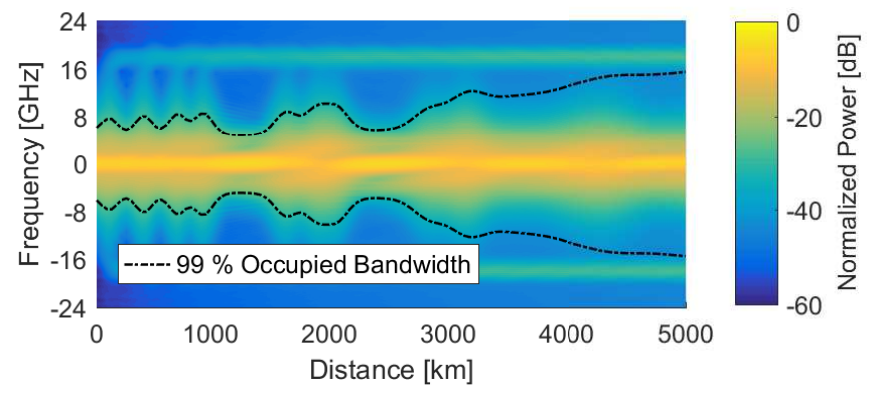

Fig. 2. The evolution of the power spectral density of a train of soliton pulses with the transmission distance and the bandwidth containing $99 \%$ of its power (dashed).
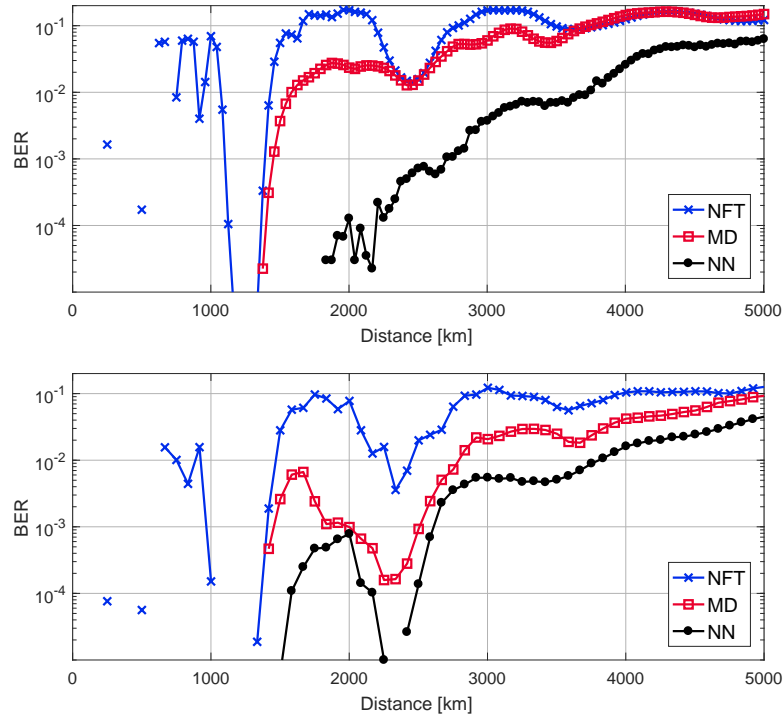

Fig. 3. BER vs transmitted distance for NFT, MD and NN receivers, single (a) and dual (b) polarization.

dual (b) polarization transmissions are shown. Further, for single polarization the performance of the $\mathrm{NN}$ detection scheme for different hyperparameters such as the number of hidden units, the sampling rate and the training data size are shown on the left in Fig. 4. Further, the hyperparameters are optimized at two transmission lengths, $2001.6 \mathrm{~km}$ and $3002.4 \mathrm{~km} \mathrm{(48}$ and 72 spans) as shown on the right in Fig. 4.

\section{B. NFT, NN and $M D$ receivers}

As NFDM communication systems are based on the lossless NLSE, transmission of thereof derived solitons suffers from attenuation and noise of real fibers and amplifiers. Further, the detection scheme applying the NFT assumes that the received pulses are stable and within their time slot. However, with increasing transmission distance the solitons gradually lose their stability until they rigorously disperse as indicated in Fig. 1 (b). This leads to a performance degradation as shown in Fig. 3. Under the chosen simulation parameters the performance already suffers at around $1000 \mathrm{~km}$, since the NFT receiver wrongly assumes to detect undistorted solitons. Moreover, the distribution of the accumulated distortion through the link is not i.i.d. Gaussian distributed, otherwise the MD receiver would be optimal and perform no worse than the NN receiver [10]. The NN receiver, trained to model the distribution of the distortions, outperforms the other receivers and allows transmission of almost $3000 \mathrm{~km}$ with a BER below $10^{-3}$. An important beneficial factor for the $\mathrm{NN}$ is that the solitons are separable in time throughout the whole transmission. This means, neighbouring solitons do not interact during transmission, such that there is no intersymbol interference or other memory effects for the NN to consider.

\section{NN receiver hyperparameters}

The NN receiver is dependent on hyperparameters such as the amount of training data, number of inputs and hidden 

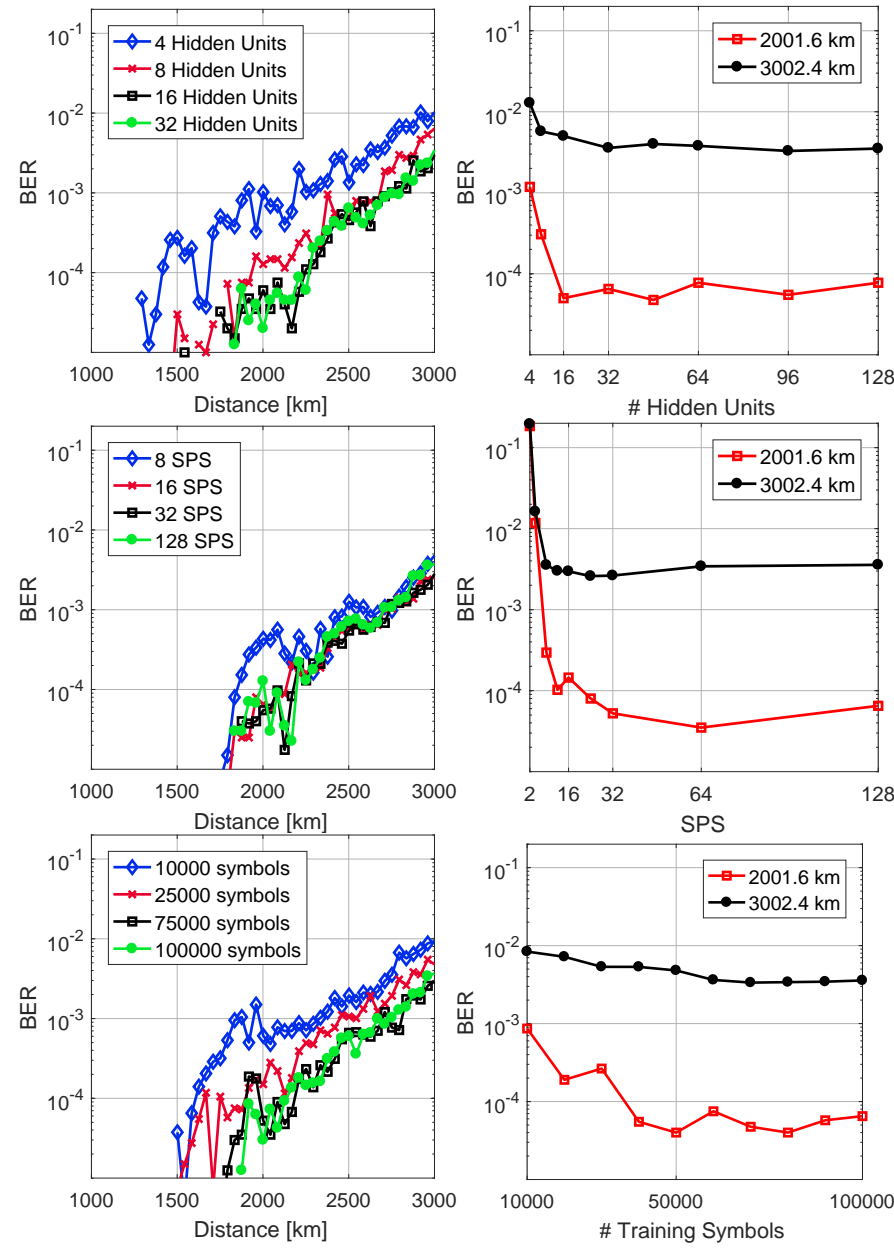

Fig. 4. NN receiver BER performance for different numbers of hidden units, numbers of SPS and sizes of the training dataset.

units. They determine the complexity of the $\mathrm{NN}$ and its potential performance. Choosing optimal hyperparameter is a multidimensional optimization problem and often solved via a grid search in the hyperparameter space [8]. Performing a grid search for every NN trained in this work is infeasible. Instead, the hyperparameters are swept independently to indicate their impact and the potential NN complexities. The complexity of an $\mathrm{NN}$ is usually given by the number of free parameters and hence by the number of hidden, input and outputs units, whereas here the number of input units is determined by the number of SPS and the number of output units by $N$. More free parameters empower an NN to learn more complex patterns from data but at the same time increase the risk of overfitting to noise. As shown in Fig. 4 (a), 16 hidden units are sufficient to model the data with an $\mathrm{NN}$, with 8 hidden units the performance is degraded and no noticeable performance improvement is achieved with 32 or more hidden units. Further, the bandwidth of the NFDM signal has no sharp bandlimit, Fig. 2, thus reducing the sampling rate is a trade-off between providing more information to the $\mathrm{NN}$ and the complexity of its input layer. Depending on the transmission distance, sampling rates of 16 to 32 are sufficient to capture the information provided by the signal, Fig. 4 (b).
The performance of the $\mathrm{NN}$ is also dependent on the amount of data used explicitly for training. Evidently from Fig. 4 (c), more data leads to better performance. In other words, the $\mathrm{NN}$ inherently models a posterior probability distribution, more data provides more evidence and thus a better model. Acquiring more data is the easiest and straight forward way of improving the NN performance [8]. The results in Fig. 4 (c) show that more than 50,000-75,000 symbols for training will yield negligible improvement in performance.

\section{CONCLUSION}

This paper offers a viable alternative receiver for NFDM optical communication systems. It outperforms the standard NFT based receiver and an MD metric receiver by using an $\mathrm{NN}$ of feasible complexity. Effects of losses and noise in NFDM systems cause distortions that the NFT receiver is not equipped for. The NN handles such impairments by learning the distortion characteristics from previous transmissions and thus is highly adaptable to the system configuration. The results obtained present a performance benchmark for future NFT receiver algorithms.

\section{ACKNOWLEDGMENT}

This work was financially supported by Keysight Technologies (Germany, Böblingen), Villum Foundation Young Investigator and by the Marie Curie Actions through ICONE Project (no. 608099).

\section{REFERENCES}

[1] R.-J. Essiambre, G. Kramer, P. J. Winzer, G. J. Foschini, and B. Goebel, "Capacity limits of optical fiber networks," Journal of Lightwave Technology, vol. 28, no. 4, pp. 662-701, 2010.

[2] A. Shabat and V. Zakharov, "Exact theory of two-dimensional selffocusing and one-dimensional self-modulation of waves in nonlinear media," Soviet physics JETP, vol. 34, no. 1, p. 62, 1972.

[3] M. I. Yousefi and F. R. Kschischang, "Information transmission using the nonlinear Fourier transform, Part III: Spectrum modulation," IEEE Transactions on Information Theory, vol. 60, no. 7, pp. 4346-4369, 2014.

[4] S. K. Turitsyn, J. E. Prilepsky, S. T. Le, S. Wahls, L. L. Frumin, M. Kamalian, and S. A. Derevyanko, "Nonlinear Fourier transform for optical data processing and transmission: advances and perspectives," Optica, vol. 4, no. 3, pp. 307-322, 2017.

[5] Z. Dong, S. Hari, T. Gui, K. Zhong, M. Yousefi, C. Lu, P.-K. A. Wai, F. Kschischang, and A. Lau, "Nonlinear frequency division multiplexed transmissions based on NFT," IEEE Photonics Techn. Lett., vol. 27, no. 15 , pp. 1621-1623, 2015.

[6] S. T. Le, J. E. Prilepsky, and S. K. Turitsyn, "Nonlinear inverse synthesis technique for optical links with lumped amplification," Optics express, vol. 23, no. 7, pp. 8317-8328, 2015.

[7] T. Gui, T. H. Chan, C. Lu, A. P. T. Lau, and P.-K. A. Wai, "Alternative decoding methods for optical communications based on nonlinear fourier transform," Journal of Lightwave Technology, vol. 35, no. 9, pp. 15421550, 2017.

[8] I. Goodfellow, Y. Bengio, and A. Courville, Deep learning. MIT press, 2016.

[9] H. Buelow, V. Aref, and W. Idler, "Transmission of waveforms determined by 7 eigenvalues with psk-modulated spectral amplitudes," in ECOC 2016; 42nd European Conference on Optical Communication; Proceedings of. VDE, 2016, pp. 1-3.

[10] J. G. Proakis, Digital communications, 1995.

[11] S. Gaiarin, A. M. Perego, E. P. da Silva, F. Da Ros, and D. Zibar, "Dualpolarization nonlinear Fourier transform-based optical communication system," Optica, vol. 5, no. 3, pp. 263-270, 2018. 